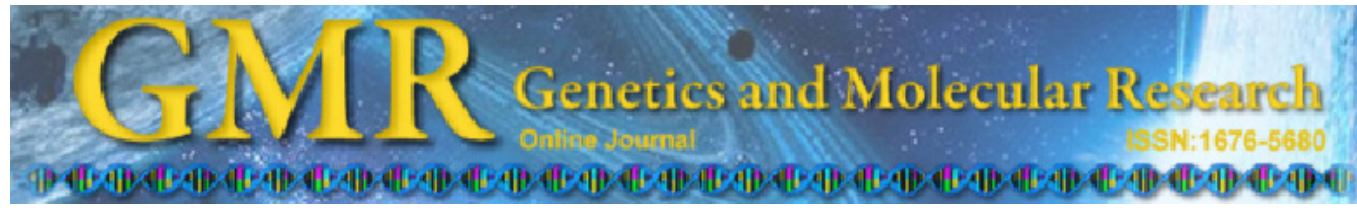

\title{
Lack of association between Cyclin D1 gene G870A polymorphism and esophageal cancer: evidence from a meta-analysis
}

\author{
W. Cai ${ }^{1 *}$, Z.T. Wang ${ }^{2 *}$, J. Zhong ${ }^{2}$ and Y. Zhang ${ }^{2,3}$ \\ ${ }^{1}$ Department of General Surgery, School of Medicine, Ruijin Hospital, \\ Shanghai Jiaotong University, Shanghai, China \\ ${ }^{2}$ Department of Gastroenterology, School of Medicine, Ruijin Hospital, \\ Shanghai Jiaotong University, Shanghai, China \\ ${ }^{3}$ Department of Pediatric Gastroenterology, School of Medicine, \\ Ruijin Hospital, Shanghai Jiaotong University, Shanghai, China \\ *These authors contributed equally to this study. \\ Corresponding author: Y. Zhang \\ E-mail: cherietoto@hotmail.com
}

Genet. Mol. Res. 12 (4): 6636-6645 (2013)

Received July 23, 2012

Accepted January 20, 2013

Published April 26, 2013

DOI http://dx.doi.org/10.4238/2013.April.26.1

\begin{abstract}
The association between the Cyclin D1 gene (CCND1) G870A polymorphism and esophageal cancer has been widely evaluated, with conflicting results. As meta-analysis is a reliable approach to resolving discrepancies, we aimed to evaluate this association. Data were available from 9 study populations incorporating 1898 cases and 3046 controls. Overall, the allelic/genotypic association between the G870A polymorphism and esophageal cancer was nonsignificant [for allele: odds ratio $(\mathrm{OR})=1.14,95 \%$ confidence interval $(95 \% \mathrm{CI})$ $=0.94-1.38, \mathrm{P}=0.184$; for genotype homozygous comparison: $\mathrm{OR}=$ $1.36,95 \% \mathrm{CI}=0.90-2.06, \mathrm{P}=0.140$; for dominant model: $\mathrm{OR}=1.24$, $95 \% \mathrm{CI}=0.88-1.75, \mathrm{P}=0.222$; for recessive model: $\mathrm{OR}=1.13,95 \% \mathrm{CI}$ $=0.90-1.43, \mathrm{P}=0.292]$. Moreover, subgroup analyses according to study designs, geographic areas, types of esophageal cancer, genotyping methods, and ethnicities failed to demonstrate a significant association between this polymorphism and esophageal cancer. In addition, there was significant publication bias as reflected by funnel plots and the
\end{abstract}


Egger test $(\mathrm{P}=0.042)$. Taken together, our results suggest that the CCND1 G870A polymorphism might not be a potential candidate for predicting esophageal cancer risk.

Key words: Esophageal cancer; CCND1 gene; Polymorphism; Meta-analysis; Genetic association

\section{INTRODUCTION}

Esophageal cancer, with a 5-year survival rate below $20 \%$, is one of the most common and most deadly malignancies worldwide (Jemal et al., 2008). Although the mechanisms of esophageal carcinogenesis are not well understood, it is generally accepted that the development of esophageal cancer is a complex, multistep, and multifactorial process involving a variety of risk factors. Specifically, smoking, drinking, micronutrient deficiency, and exposure to dietary carcinogens have been reported to be the main contributors to this disease. However, only a small portion of at-risk individuals exposed to the above factors will develop esophageal cancer, and most patients do not carry these known risk factors (Hiyama et al., 2007), suggesting genetic involvement in esophageal carcinogenesis.

Cyclin D1, also known as CCND1, is located on chromosome 11q13 and is a key regulator of the G1 phase of the cell cycle (Fu et al., 2004). CCND1 binds to and activates its kinase partners CDK4 and CDK6, which results in the phosphorylation of the retinoblastoma protein and further affects the transcription of genes that promote progression to the S-phase of the cell cycle (Mallya and Arnold, 2000). Experimental models showed that upregulation of CCND1 expression enhanced the metastatic efficiency of esophageal cancer (Zhou et al., 2009), supporting the notion that $C C N D 1$ plays a pivotal role in the development of esophageal cancer.

Many polymorphisms have been identified in CCND1. A common functional polymorphism, G870A (rs603965), which increased the frequency of alternative splicing and encoded a protein with an altered C-terminal domain and increased the stability or half-life of the protein, has garnered wide attention. It was proposed that DNA-damaged cells in individuals with the A allele may bypass the G1/S checkpoint, leading to an increased proportion of cells with DNA damage and genetic alterations (Betticher et al., 1995). Meanwhile, epidemiological studies have reported an association between the CCND1 A/A genotype and increased risk of various cancers, including cervical (Jeon et al., 2005; Satinder et al., 2008; Thakur et al., 2009) and colorectal cancer (Ho-Pun-Cheung et al., 2007; Talseth et al., 2008; Tan et al., 2008). Although some studies have attempted to link this polymorphism with esophageal cancer, the results are often not reproducible. Generally, replication failure might result from genetic heterogeneity across different races or ethnicities, as well as individually underpowered studies. To address this issue, we investigated whether the CCND1 G870A polymorphism is associated with esophageal cancer by meta-analysis and assessed whether this polymorphism shows genetic heterogeneity across different study designs, geographic areas, types of esophageal cancer, genotyping methods, or ethnicities.

\section{MATERIAL AND METHODS}

\section{Literature search}

We searched the MEDLINE, EMBASE, and Web of Science engines for studies 
published before March 7, 2012. The key words used for searching were "Cyclin D1" or "CCND1" and "esophageal cancer" along with "polymorphism".

We also checked the reference lists of all retrieved articles to ensure the comprehensiveness of this meta-analysis. If articles involved more than 1 geographic or ethnic heterogeneous group, we showed them separately. If more than 1 article shared the same sample, we removed the article with smaller sample size. Articles written in English and studies performed in humans were identified.

\section{Inclusion and exclusion criteria}

Articles were included if they evaluated the CCND1 gene G870A polymorphism and esophageal cancer risk; if they were conducted in a case-control, nested case-control, or cross-sectional design; and if they provided sufficient information regarding genotype distributions between both cases and controls. Meanwhile, we only focused on esophageal cancer rather than other 2nd neoplasms. We excluded case reports or series, editorials, review articles, and non-English articles.

\section{Extracted information}

From each qualified articles, two authors (W.C. and Z.T.W.) independently drew the following information: 1st author's last name, year of publication, ethnicity of the population studied, study design, number of subjects in each category, baseline characteristics of the study populations, and the number of persons with different genotypes in cases and controls. We resolved discrepancies by discussion until a consensus was reached.

\section{Statistical analysis}

In this meta-analysis, we used the allelic (870A vs 870G), homozygous (870AA vs 870GG), dominant (870AA plus 870GA $v s$ 870GG), and recessive models (870AA vs 870GG plus $870 \mathrm{GA}$ ). Hardy-Weinberg equilibrium was calculated by the $\chi^{2}$ test.

The fixed-effect model was used if between-study heterogeneity was absent ( $I^{2}$ statistics) and the random-effect model was used otherwise (Higgins et al., 2003). In this metaanalysis, only the random-effect model was applied since within a fixed-effect model, only sampling error contributes to the differences between the observed effect-size estimates across individual studies (Cohn and Becker, 2003; Borenstein et al., 2009). Between-study heterogeneity was quantified by the inconsistency index $I^{2}$ statistic, which ranged from 0 to $100 \%$. The $I^{2}$ statistic was documented for the percentage of the observed between-study variability due to heterogeneity rather than chance, with higher values of this index suggesting the existence of heterogeneity (Higgins and Thompson, 2002; Higgins et al., 2003). If between-study heterogeneity was significant, we examined the study characteristics that could stratify the studies into subgroups with homogeneous effects.

Publication bias was tested by funnel plots and the Egger test. The Egger test can detect funnel plot asymmetry by determining whether the intercept deviates significantly from 0 in a regression of the standardized effect estimates against their precision.

Significance was judged at $\mathrm{P}<0.1$ for the $I^{2}$ statistic and the Egger test. We managed the data and performed statistical analyses by using the STATA software (version 11.0 for Windows; StataCorp, College Station, TX, USA). 


\section{RESULTS}

\section{Baseline characteristics}

After an extensive search, a total of 12 studies were collected based on our inclusion/ exclusion criteria. If more than 1 geographical or ethnic group was included in the same study, then data from different populations were extracted. Therefore, 8 studies, including 9 populations (Yu et al., 2003; Zhang et al., 2003; Casson et al., 2005; Geddert et al., 2005; Jain et al., 2007; Akbari et al., 2009; Liu et al., 2010; Hussain et al., 2011) with 1898 patients with esophageal cancer and 3046 controls were finally identified: 4 populations were from East Asia [2 Chinese (Yu et al., 2003; Zhang et al., 2003) and 2 from India (Jain et al., 2007; Hussain et al., 2011)], 2 were from West Asia (Akbari et al., 2009), and 3 included Caucasians (Casson et al., 2005; Geddert et al., 2005; Liu et al., 2010). A flow diagram schematizing the process of selected and excluded articles with specific reasons for each is presented in Figure 1.

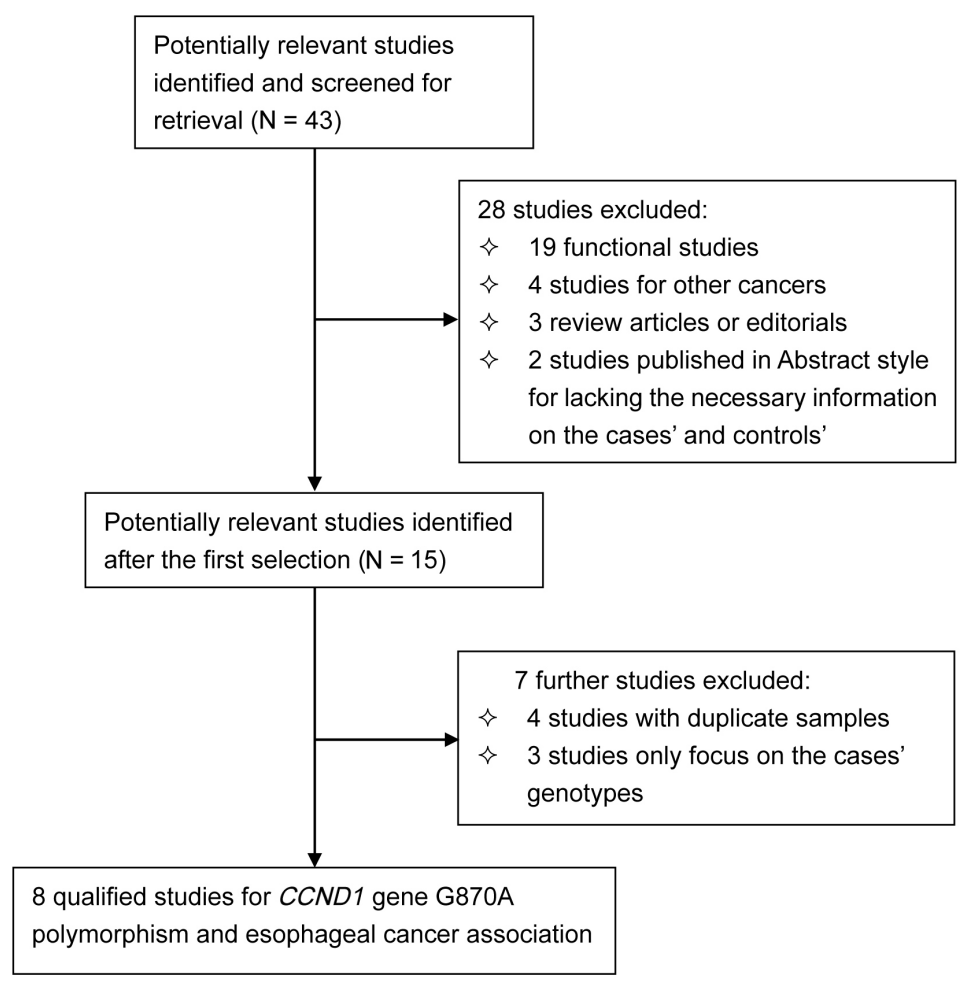

Figure 1. Flow diagram of search strategy and study selection.

With regard to the study design, 3 of these studies were population based (Yu et al., 2003; Zhang et al., 2003; Akbari et al., 2009), and 5 employed a hospital-based design (Casson et al., 2005; Geddert et al., 2005; Jain et al., 2007; Liu et al., 2010; Hussain et al., 2011). The baseline characteristics of all eligible studies are summarized in Table 1. 
W. Cai et al.

6640

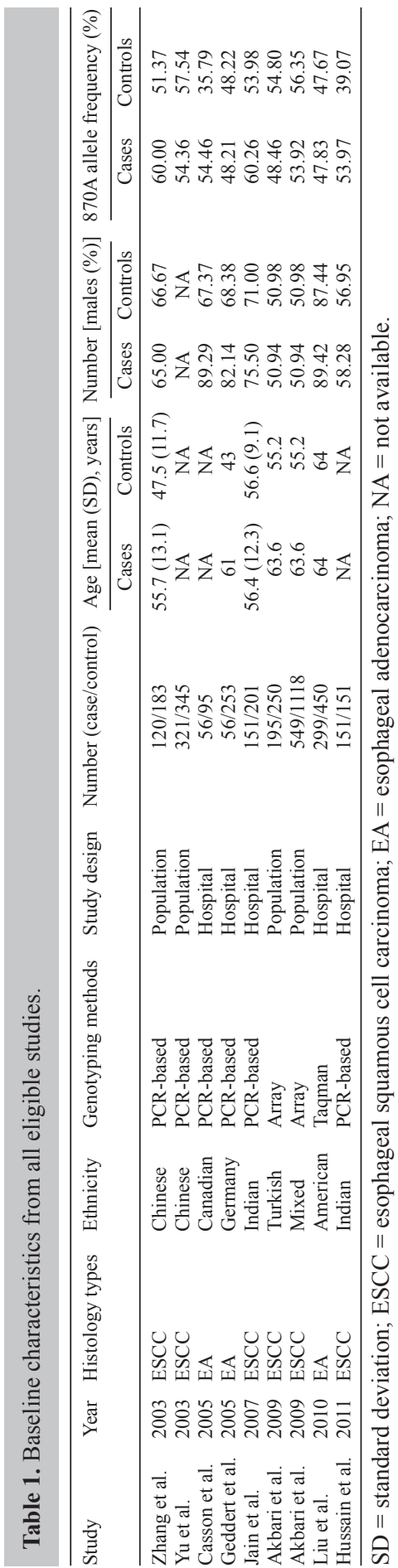

Genetics and Molecular Research 12 (4): 6636-6645 (2013) 
The frequencies of the $C C N D 1$ gene $+870 \mathrm{~A}$ allele in patients ranged from 47.83 to $60.26 \%$, and that of controls varied from 35.79 to $57.54 \%$. No deviations from Hardy-Weinberg equilibrium were observed in the genotype distributions of controls at the significance level of 0.05 .

\section{Genetic association}

The combined results based on all studies showed that there was no statistically significant link between the CCND1 G870A polymorphism and esophageal cancer susceptibility in the allele model [odds ratio $(\mathrm{OR})=1.14,95 \%$ confidence interval $(95 \% \mathrm{CI})=0.94-1.38, \mathrm{P}$ $=0.184]$. Since the test for heterogeneity among the studies was significant $\left(\mathrm{P}<0.0005, I^{2}=\right.$ $78.1 \%$; Figure 2), the random-effect model was conducted. Lack of significance persisted in homozygous models for comparison of 870AA with $870 \mathrm{GG}(\mathrm{OR}=1.36,95 \% \mathrm{CI}=0.90-2.06$, $\mathrm{P}=0.140)$, as well as in dominant $(\mathrm{OR}=1.24,95 \% \mathrm{CI}=0.88-1.75, \mathrm{P}=0.222)$ and recessive $(\mathrm{OR}=1.13,95 \% \mathrm{CI}=0.90-1.43, \mathrm{P}=0.292)$ models.

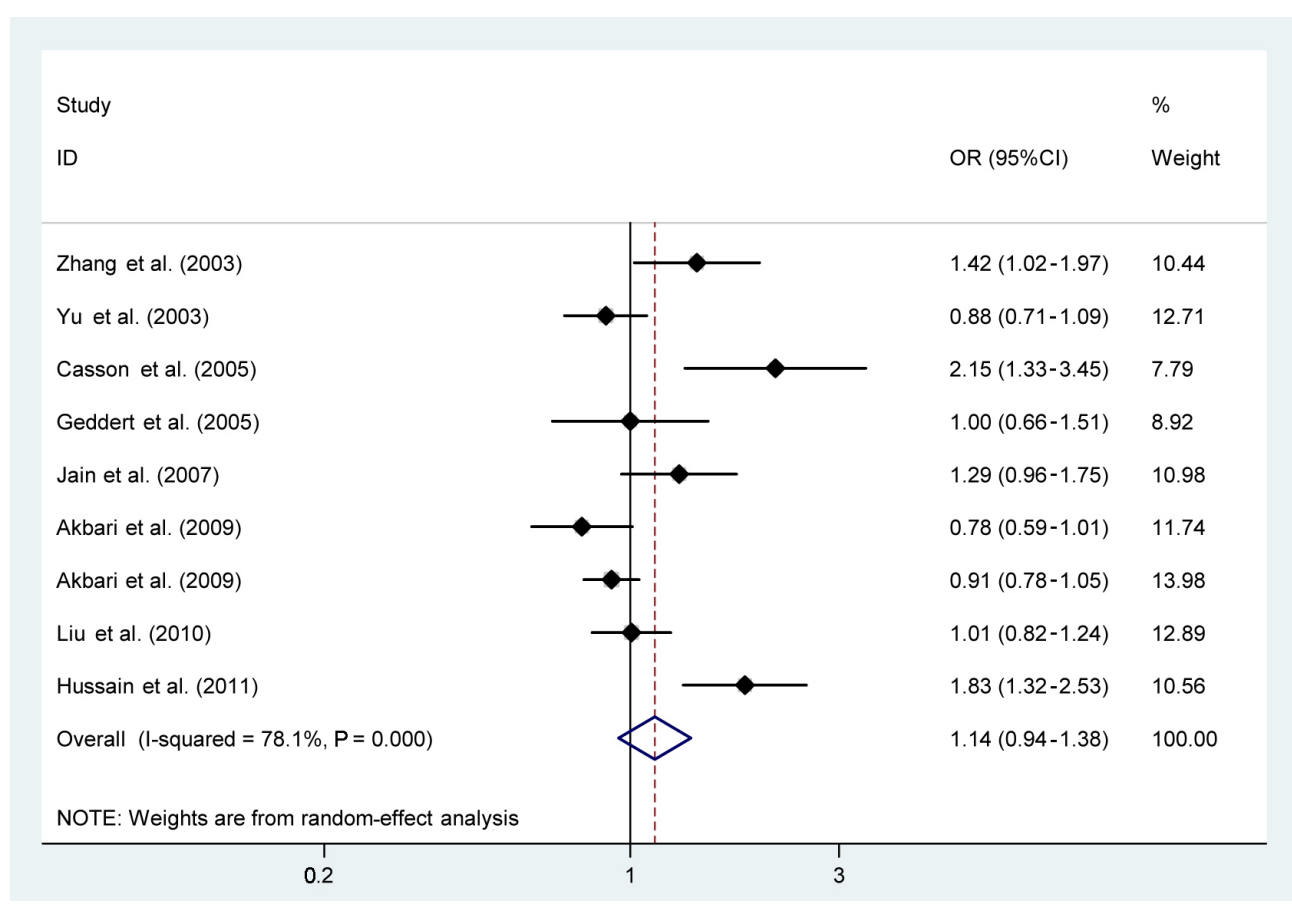

Figure 2. Contrast of the $C C N D 1$ gene $870 \mathrm{~A}$ allele versus the $870 \mathrm{G}$ allele. The combined results based on all studies showed that there was no statistically significant link between the CCND1 G870A polymorphism and esophageal cancer susceptibility in allele model. Odds ratio $(\mathrm{OR})=1.14,95 \%$ confidence interval $(95 \% \mathrm{CI})=0.94-1.38$.

\section{Subgroup analyses}

Considering that the study design, the geographic difference, the type of esophageal cancer [esophageal adenocarcinoma (EA) or esophageal squamous cell carcinoma (ESCC)], 
and the method of genotyping might bias the overall association results, we conducted separate analyses according to these factors.

In view of the study design, no obvious association existed in the population-based subgroup, although a significant association between the CCND1 A870G polymorphism and esophageal cancer risk was observed for the allele model $(\mathrm{OR}=1.35,95 \% \mathrm{CI}=1.01-1.80, \mathrm{P}=$ $0.04)$ and the homozygous genotype model $(\mathrm{OR}=1.95,95 \% \mathrm{CI}=1.02-3.75, \mathrm{P}=0.044)$ in the hospital-based subgroup.

When stratifying by the genotyping method, no obvious association existed in either the Taqman or Chip subgroup, while a significant increased risk was found in the PCR-based subgroup for the allele model $(\mathrm{OR}=1.33,95 \% \mathrm{CI}=1.01-1.76, \mathrm{P}=0.045)$. Similarly, there was a statistically significant increased risk in the PCR-based subgroup for the homozygous genotype model $(\mathrm{OR}=1.98,95 \% \mathrm{CI}=1.02-3.85, \mathrm{P}=0.043)$, as well as in the recessive model $(\mathrm{OR}=1.42,95 \% \mathrm{CI}=1.00-2.02, \mathrm{P}=0.047)$.

In the subgroup analysis based on geographic area, we still observed no material changes except in West Asian studies. For example, in populations from West Asia, the 870A allele and 870AA homozygous genotype had 13 and 23\% reduced risk of esophageal cancer (for the $870 \mathrm{~A}$ allele: $\mathrm{OR}=0.87,95 \% \mathrm{CI}=0.77-0.99, \mathrm{P}=0.041$; for the 870AA genotype: $\mathrm{OR}$ $=0.77,95 \% \mathrm{CI}=0.60-0.99, \mathrm{P}=0.043)$, respectively.

In the subgroup meta-analysis based on the type of esophageal cancer, nearly no changes in ORs were observed in the ESCC and EA subgroups for CCND1 A870G polymorphisms.

\section{Publication bias}

As reflected by the funnel plot (Figure 3) and the Egger test, there was significant publication bias existing for the $C C N D 1$ gene $\mathrm{A} 870 \mathrm{G}$ polymorphism $(\mathrm{t}=2.49, \mathrm{P}=0.042)$.

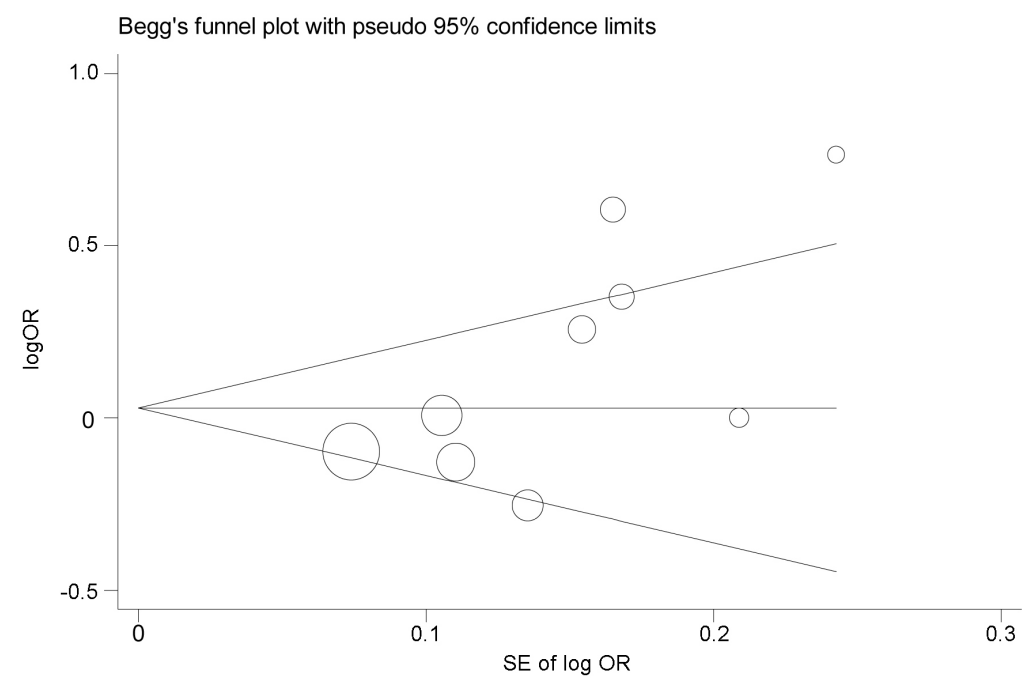

Figure 3. Begg's funnel plot of publication bias test for the CCND1 A870G polymorphism. As reflected by the funnel plot, there was significant publication bias exiting for the $C C N D 1$ gene $\mathrm{A} 870 \mathrm{G}$ polymorphism. $\mathrm{SE}=$ standard error. $\mathrm{OR}=$ odds ratio. 


\section{DISCUSSION}

Although some statistical biases could not be eliminated and the between-study heterogeneity was alarming, our results suggest that the CCND1 A870G polymorphism is not significantly associated with esophageal cancer in general populations. This study, including 4944 subjects from 9 populations, to our knowledge, is the 1st meta-analysis examining the relationship between the CCND1 A870G polymorphism and occurrence of esophageal cancer. This result requires further investigation, not only due to the relatively small sample size, but also because of genetic heterogeneity and the differences in the study design, genotyping method, and esophageal cancer type, which were identified as potentially significant sources of between-study heterogeneity in this study.

Firstly, genetic heterogeneity is an inevitable problem in any disease identification strategy (Hemminki et al., 2006). In our analyses by geographic areas, the CCND1 A870G polymorphism showed significant heterogeneity in esophageal cancer across different subgroups, with the 870A allele and the 870AA genotype in West Asia presenting 13 and 23\% reduced risk, respectively. However, we could not detect any significant esophageal cancer risk variation for all genetic models in East Asians or Caucasians. Different genetic backgrounds may cause this discrepancy or different populations may have different linkage disequilibrium patterns. A polymorphism may be in close linkage with another nearby causal variant in one ethnic population but not in another (Yu et al., 2010). Thus, it is reasonable to hypothesize that the CCND1 A870G polymorphism might be in close linkage with different nearby causal variants in different populations. Moreover, this polymorphism might have a pleiotropic role in the pathogenesis of esophageal cancer or might interact with other genetic and environmental factors. However, considering the relatively small sample sizes in this study, we suggest that confirmation in large, well-designed studies is critical.

Secondly, study design and genotyping method might also be significant contributors to the CCND1 A870G polymorphism and esophageal cancer risk. With regard to study design, although allele and homozygous genotype comparison of the A870G polymorphism generated a marginally significant association in hospital-based studies, we ran the risk of overestimating the magnitude of this association in view of the striking weaknesses of this type of design, such as population stratification and admixture. Contrastingly, no positive signal was identified in population-based studies, reinforcing the quality of our conclusion. For the genotyping method, a marginal association was noted with the PCR-based method, which was susceptible to genotypic misclassification errors. Likewise, a negative association was preserved in the Chip or Taqman method. Therefore, to obtain convincing evidence, well-designed studies using less error-prone methods are encouraged.

Thirdly, EA and ESCC are the 2 main histological types of esophageal cancer and have great differences in etiology and tumor biology (van Baal et al., 2008). For example, EA is quite common in Western countries, and it is widely believed that EA arises from Barrett esophagus, an acquired condition in which the normal esophageal squamous epithelium is replaced by a metaplastic columnar cell-lined epithelium (Williams et al., 2006). However, ESCC is the major subtype in the Asia-Pacific countries, and its development is reportedly attributed to smoking, alcohol consumption, and betel quid chewing (Chung et al., 2010). In view of this geographic distribution difference, we subgrouped studies according to the types of esophageal cancer and found that the magnitude of the association between the CCND1 
A870G polymorphism and esophageal cancer was augmented in the EA group relative to the ESCC group across all genetic comparisons except in the dominant model (Table 2), although the pooled associations lacked statistical significance. Considering the relatively small sample sizes in each subgroup, additional research within the framework of genetics and biology is needed in various types of esophageal cancer.

\begin{tabular}{|c|c|c|c|c|c|c|c|c|c|}
\hline \multirow[t]{2}{*}{ Subgroup } & \multirow[t]{2}{*}{ Study number } & \multicolumn{2}{|l|}{$\mathrm{A} v s \mathrm{G}$} & \multicolumn{2}{|l|}{$\mathrm{AA} v s \mathrm{GG}$} & \multicolumn{2}{|l|}{ Dominant } & \multicolumn{2}{|l|}{ Recessive } \\
\hline & & OR $(95 \% \mathrm{CI})$ & $\mathrm{P}$ & OR $(95 \% \mathrm{CI})$ & $\mathrm{P}$ & OR $(95 \% \mathrm{CI})$ & $\mathrm{P}$ & OR $(95 \% \mathrm{CI})$ & $P$ \\
\hline \multicolumn{10}{|l|}{ Geographic area } \\
\hline East Asians & 4 & $1.29(0.93-1.80)$ & 0.128 & $1.85(0.84-4.09)$ & 0.129 & $1.74(0.79-3.81)$ & 0.169 & $1.22(0.93-1.60)$ & 0.159 \\
\hline West Asians & 2 & $0.87(0.77-0.99)$ & 0.041 & $0.77(0.60-0.99)$ & 0.043 & $0.80(0.60-1.07)$ & 0.128 & $0.85(0.70-1.04)$ & 0.117 \\
\hline Caucasians & 3 & $1.25(0.81-1.91)$ & 0.310 & $1.67(0.64-4.34)$ & 0.291 & $1.18(0.77-1.81)$ & 0.443 & $1.59(0.68-3.70)$ & 0.280 \\
\hline \multicolumn{10}{|l|}{ Study design } \\
\hline Population & 4 & $0.94(0.78-1.14)$ & 0.543 & $0.92(0.59-1.42)$ & 0.696 & $0.94(0.63-1.40)$ & 0.754 & $0.91(0.77-1.06)$ & 0.226 \\
\hline Hospital & 5 & $1.35(1.01-1.80)$ & 0.040 & $1.95(1.02-3.75)$ & 0.044 & $1.56(0.93-2.62)$ & 0.092 & $1.48(0.96-2.28)$ & 0.078 \\
\hline \multicolumn{10}{|c|}{ 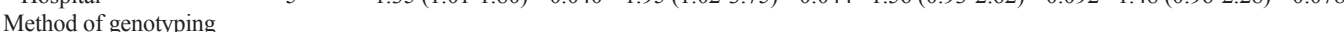 } \\
\hline PCR-based & 6 & $1.33(1.01-1.76)$ & 0.045 & $1.98(1.02-3.85)$ & 0.043 & $1.58(0.89-2.80)$ & 0.117 & $1.42(1.00-2.02)$ & 0.047 \\
\hline Chip or Taqman & 3 & $0.91(0.81-1.02)$ & 0.111 & $0.83(0.66-1.03)$ & 0.084 & $0.89(0.68-1.15)$ & 0.361 & $0.87(0.73-1.03)$ & 0.103 \\
\hline \multicolumn{10}{|l|}{ Type of EC } \\
\hline ESCC & 6 & $1.10(0.87-1.40)$ & 0.409 & $1.28(0.77-2.14)$ & 0.336 & $1.27(0.78-2.07)$ & 0.330 & $1.04(0.84-1.29)$ & 0.713 \\
\hline EA & 3 & $1.25(0.81-1.91)$ & 0.310 & $1.67(0.64-4.34)$ & 0.291 & $1.18(0.77-1.81)$ & 0.443 & $1.59(0.68-3.70)$ & 0.280 \\
\hline
\end{tabular}

$\mathrm{OR}=$ odds ratio $; 95 \% \mathrm{CI}=95 \%$ confidence interval; $\mathrm{EC}=$ esophageal cancer; $\mathrm{ESCC}=$ esophageal squamous cell carcinoma; EA = esophageal adenocarcinoma.

Lastly, some limitations of this meta-analysis should be addressed. First, only articles written in English were identified in this meta-analysis, which may be the major cause of the high probability of publication bias. Second, most of the studies included were limited by the small sample size. Moreover, the population from 8 studies was not uniform. As in other investigations, the source of heterogeneity may include geographic area, the study design, the geographic area, the type of esophageal cancer (EA or ESCC), as well as the method of genotyping used. Third, the single-locus-based nature of this meta-analysis precluded the possibility of gene-gene and gene-environment interactions, as well as haplotype-based effects, suggesting that additional studies assessing these aspects will be necessary. Furthermore, we only centered on the CCND1 A870G polymorphism and did not evaluate other genes or polymorphisms. It seems likely that the A870G polymorphism individually makes a moderate contribution to risk prediction in esophageal cancer patients, although whether this polymorphism integrated with other risk factors will enhance the predictive power requires additional research. Thus, we must refrain from drawing a firm conclusion until large, well-performed studies confirm or refute our results.

Taken together, we expanded previous individually underpowered studies regarding esophageal cancer risk by suggesting that no obvious association was found between CCND1 A870G and esophageal cancer susceptibility. In addition, our observations raise the question of a potential heterogeneous effect of A870G across different ethnic populations. Nevertheless, for practical reasons, we hope that this study will not remain just another endpoint of research, and instead will represent a beginning to establish the background data for further investigation of the association between the CCND1 gene and esophageal cancer. 


\section{REFERENCES}

Akbari MR, Malekzadeh R, Shakeri R, Nasrollahzadeh D, et al. (2009). Candidate gene association study of esophageal squamous cell carcinoma in a high-risk region in Iran. Cancer Res. 69: 7994-8000.

Betticher DC, Thatcher N, Altermatt HJ, Hoban P, et al. (1995). Alternate splicing produces a novel cyclin D1 transcript. Oncogene 11: 1005-1011.

Borenstein M, Hedges LV, Higgins JPT and Rothstein HR (2009). Introduction to Meta-Analysis. 1st edn. Wiley Publishers, Chichester.

Casson AG, Zheng Z, Evans SC, Geldenhuys L, et al. (2005). Cyclin D1 polymorphism (G870A) and risk for esophageal adenocarcinoma. Cancer 104: 730-739.

Chung CS, Lee YC, Wang CP, Ko JY, et al. (2010). Secondary prevention of esophageal squamous cell carcinoma in areas where smoking, alcohol, and betel quid chewing are prevalent. J. Formos Med. Assoc. 109: 408-421.

Cohn LD and Becker BJ (2003). How meta-analysis increases statistical power. Psychol. Methods 8: 243-253.

Fu M, Wang C, Li Z, Sakamaki T, et al. (2004). Minireview: Cyclin D1: normal and abnormal functions. Endocrinology 145: 5439-5447.

Geddert H, Kiel S, Zotz RB, Zhang J, et al. (2005). Polymorphism of p16 INK4A and cyclin D1 in adenocarcinomas of the upper gastrointestinal tract. J. Cancer Res. Clin. Oncol. 131: 803-808.

Hemminki K, Lorenzo Bermejo J and Forsti A (2006). The balance between heritable and environmental aetiology of human disease. Nat. Rev. Genet. 7: 958-965.

Higgins JP and Thompson SG (2002). Quantifying heterogeneity in a meta-analysis. Stat. Med. 21: 1539-1558.

Higgins JP, Thompson SG, Deeks JJ and Altman DG (2003). Measuring inconsistency in meta-analyses. BMJ 327: 557-560.

Hiyama T, Yoshihara M, Tanaka S and Chayama K (2007). Genetic polymorphisms and esophageal cancer risk. Int. J. Cancer 121: 1643-1658.

Ho-Pun-Cheung A, Assenat E, Thezenas S, Bibeau F, et al. (2007). Cyclin D1 gene G870A polymorphism predicts response to neoadjuvant radiotherapy and prognosis in rectal cancer. Int. J. Radiat. Oncol. Biol. Phys. 68: 1094-1101.

Hussain S, M Y, Thakur N, Salam I, et al. (2011). Association of cyclin D1 gene polymorphisms with risk of esophageal squamous cell carcinoma in Kashmir Valley: a high risk area. Mol. Carcinog. 50: 487-498.

Jain M, Kumar S, Lal P, Tiwari A, et al. (2007). Role of BCL2 (ala43thr), CCND1 (G870A) and FAS (A-670G) polymorphisms in modulating the risk of developing esophageal cancer. Cancer Detect. Prev. 31: 225-232.

Jemal A, Siegel R, Ward E, Hao Y, et al. (2008). Cancer statistics, 2008. CA Cancer J. Clin. 58: 71-96.

Jeon YT, Kim JW, Song JH, Park NH, et al. (2005). Cyclin D1 G870A polymorphism and squamous cell carcinoma of the uterine cervix in Korean women. Cancer Lett. 223: 259-263.

Liu G, Cescon DW, Zhai R, Zhou W, et al. (2010). p53 Arg72Pro, MDM2 T309G and CCND1 G870A polymorphisms are not associated with susceptibility to esophageal adenocarcinoma. Dis. Esophagus 23: 36-39.

Mallya SM and Arnold A (2000). Cyclin D1 in parathyroid disease. Front Biosci. 5: D367-D371.

Satinder K, Chander SR, Pushpinder K, Indu G, et al. (2008). Cyclin Dl (G870A) polymorphism and risk of cervix cancer: a case control study in north Indian population. Mol. Cell Biochem. 315: 151-157.

Talseth BA, Ashton KA, Meldrum C, Suchy J, et al. (2008). Aurora-A and Cyclin D1 polymorphisms and the age of onset of colorectal cancer in hereditary nonpolyposis colorectal cancer. Int. J. Cancer 122: 1273-1277.

Tan XL, Nieters A, Kropp S, Hoffmeister M, et al. (2008). The association of cyclin D1 G870A and E-cadherin C-160A polymorphisms with the risk of colorectal cancer in a case control study and meta-analysis. Int. J. Cancer 122: 2573-2580.

Thakur N, Hussain S, Kohaar I, Tabassum R, et al. (2009). Genetic variant of CCND1: association with HPV-mediated cervical cancer in Indian population. Biomarkers 14: 219-225.

van Baal JW, Milana F, Rygiel AM, Sondermeijer CM, et al. (2008). A comparative analysis by SAGE of gene expression profiles of esophageal adenocarcinoma and esophageal squamous cell carcinoma. Cell Oncol. 30: 63-75.

Williams LJ, Guernsey DL and Casson AG (2006). Biomarkers in the molecular pathogenesis of esophageal (Barrett) adenocarcinoma. Curr. Oncol. 13: 33-43.

Yu C, Lu W, Tan W, Xing D, et al. (2003). Lack of association between CCND1 G870A polymorphism and risk of esophageal squamous cell carcinoma. Cancer Epidemiol. Biomarkers Prev. 12: 176.

Yu K, Zhang J, Zhang J, Dou C, et al. (2010). Methionine synthase A2756G polymorphism and cancer risk: a metaanalysis. Eur. J. Hum. Genet. 18: 370-378.

Zhang J, Li Y, Wang R, Wen D, et al. (2003). Association of cyclin D1 (G870A) polymorphism with susceptibility to esophageal and gastric cardiac carcinoma in a northern Chinese population. Int. J. Cancer 105: 281-284.

Zhou W, Ye XL, Sun ZJ, Ji XD, et al. (2009). Overexpression of degenerative spermatocyte homolog 1 up-regulates the expression of cyclin D1 and enhances metastatic efficiency in esophageal carcinoma Eca109 cells. Mol. Carcinog. 48: 886-894. 